\title{
PROPAGATION OF A BOUNDARY OF FUSION
}

\author{
by STEWART PATERSON \\ (Received 20th December, 1949)
}

1. General Theory. We consider a volume of material, divided into two regions 1 and 2. each of density $\rho$, by a moving surface $S$. On $S$ a change of phase occurs, at a definite temperature (which we may take to be zero) and with absorption or liberation of a latent heat $L$ per unit mass. If $\theta_{1}, k_{1}, K_{1}$ are the temperature, thermal conductivity and diffusivity of phase 1 , and $\theta_{2}, k_{2}, K_{2}$ corresponding quantities for phase 2 , the surface $S$ is the isothermal

$$
\theta_{1}(x, y, z, t)=0=\theta_{2}(x, y, z, t)
$$

and the boundary condition on this surface is

$$
k_{1}\left|\operatorname{grad} \theta_{1}\right|-k_{2}\left|\operatorname{grad} \theta_{2}\right|= \pm L \rho \frac{\left(\theta_{1}\right)_{t}}{\left|\operatorname{grad} \theta_{1}\right|}= \pm L \rho \frac{\left(\theta_{2}\right)_{t}}{\left|\operatorname{grad} \theta_{2}\right|} .
$$

Subscript letters denote partial differentiation.

The condition for a simple solution is thus seen at once to be that $\theta_{1}, \theta_{2}$ separately satisfy

$$
\frac{\operatorname{grad}^{2} \theta}{\theta_{t}}=\text { const. when } \theta=0 \text {. }
$$

For example, in the one-dimensional cases of linear, axially symmetrical or spherically symmetrical flow, we require

$$
\theta_{r}^{2} / \theta_{t}=\text { const. when } \theta=0 \text {, }
$$

where $r$ is the appropriate space co-ordinate.

In addition, $\theta_{1}, \theta_{2}$ must, of course, satisfy

$$
\left(\theta_{1}\right)_{t}=K_{1} \nabla^{2} \theta_{1} ; \quad\left(\theta_{2}\right)_{t}=K_{2} \nabla^{2} \theta_{2}
$$

which for the above one-dimensional cases become

$$
\left(\theta_{1}\right)_{t}=K_{1}\left[\left(\theta_{1}\right)_{r r}+\frac{n\left(\theta_{1}\right)_{r}}{r}\right] ; \quad\left(\theta_{2}\right)_{t}=K_{2}\left[\left(\theta_{2}\right)_{r r}+\frac{n\left(\theta_{2}\right)_{r}}{r}\right],
$$

where $n \equiv 0,1,2$ in the three cases, respectively.

2. Particular solutions. We consider solutions of the form

$$
\begin{aligned}
& \theta=v(\zeta), \ldots . . \\
& \zeta \equiv \phi(r) \psi(t) .
\end{aligned}
$$

where

It has been shown elsewhere that the only such solutions are :

$$
\begin{aligned}
& \text { Linear case }\left\{\begin{array}{l}
\theta=A \operatorname{erf} *\left(\frac{r+B}{2 \sqrt{K(t+E)}}\right)+D, \\
\theta=e^{A^{3} K t}\left[B e^{A}+C e^{-A r}\right]+D, \ldots \\
\theta=A r+B . \ldots \ldots \ldots \ldots \ldots \ldots \ldots \ldots \ldots \ldots \ldots
\end{array}\right. \\
& * \operatorname{erf}(x) \equiv 1-\operatorname{erfc}(x) \equiv \frac{2}{\sqrt{\pi}} \int_{0}^{x} e^{-u^{2}} d u ; E i(x) \equiv \int_{\infty}^{-x} e^{-u} d u / u \text {. }
\end{aligned}
$$




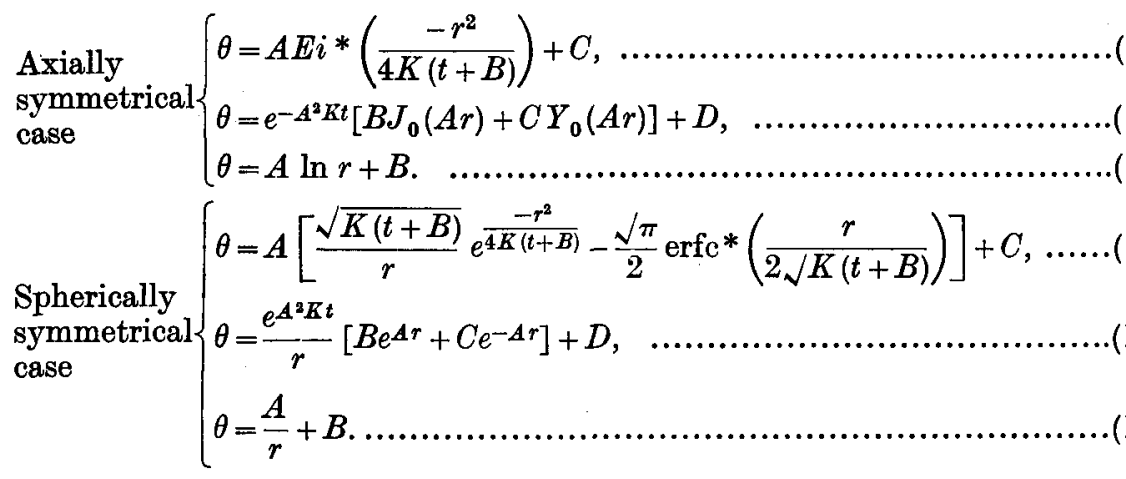

Of these we must discard the trivial solutions (11), (14) and (17); and also (12) and (16), which cannot be made to satisfy (4). (4) is, however, satisfied by (9), (12) and (15), and also by (10), if either $B$ or $C$ is zero.

Solution (10), which was mentioned by Stefan (1), defines a boundary of fusion moving with constant speed. It is of restricted interest, For example, consider the solution

$$
\begin{aligned}
& \theta_{1}=B\left[e^{-A\left(r-A K_{1} t\right)}-1\right], \quad r \geqslant A K_{1} t, \ldots . . \\
& \theta_{2}=C\left[e^{\frac{K_{1}}{K_{2}} A\left(A K_{1} t-r\right)}-1\right], \quad 0 \leqslant r \leqslant A K_{1} t .
\end{aligned}
$$

This describes the behaviour of a semi-infinite solid, whose initial temperature is

$$
\theta_{1}=B\left(e^{-A r}-1\right)
$$

and whose surface temperature at $r=0$ is

$$
\theta_{2}=C\left(e^{\frac{K_{1}^{2}}{K_{2}} A^{2 t}}-1\right)
$$

However, $B$ and $C$ must, by (2), satisfy

$$
k_{2} C / K_{2}-k_{1} B / K_{1}=L \rho
$$

(9), (12) and (15) define boundaries of fusion whose position varies linearly with the square root of the time, measured from an appropriate zero. Solutions based on (9) are, in fact, wellknown, and correspond to problems of some importance (2). (12) and (15) also yield solutions of practical interest, which do not appear to have been studied.

3. Propagation of a Boundary of Fusion from a line source of heat. Let $Q$ units of heat be generated per unit time, per unit length of the line $r=0$, in an infinite fusible solid, whose initial temperature is $-\Theta$, referred to the melting point as zero. A cylindrical boundary of fusion, $r=R(t)$ say, then advances into the solid. Let suffices 1, 2 refer to the solid and liquid phases respectively. We assume a solution :

whereupon

$$
\begin{aligned}
& \theta_{1}=-A E i\left(-r^{2} / 4 K_{1} t\right)-B, \\
& \theta_{2}=-C E i\left(-r^{2} / 4 K_{2} t\right)-D,
\end{aligned}
$$

$$
R(t)=\alpha \sqrt{t}
$$

where $\alpha$ is a constant to be determined.

Then

$$
\begin{array}{r}
A E i\left(-\alpha^{2} / 4 K_{1}\right)+B=0 \\
C E i\left(-\alpha^{2} / 4 K_{2}\right)+D=0 . \\
* \text { See footnote, p. } 42 .
\end{array}
$$


When $t=0, R=0$, and $\theta_{1}=-B$. Hence

$$
B=\Theta \text {. }
$$

Also,

$$
\frac{\partial \theta_{1}}{\partial r}=-\frac{2 A}{r} e^{-r^{2} / 4 K_{1} t} ; \quad \frac{\partial \theta_{2}}{\partial r}=-\frac{2 C}{r} e^{-r^{2} / 4 K_{2} t}
$$

Thus

$$
\underset{r \rightarrow 0}{\mathrm{~L}}\left[-2 \pi k_{2} r \frac{\partial \theta_{2}}{\partial r}\right]=4 \pi k_{2} C,
$$

so that

$$
C=\frac{Q}{4 \pi k_{2}}
$$

Again, by (2), (22) and (26),

$$
k_{2} C e^{-\alpha^{2} / 4 K_{2}}-k_{1} A e^{-\alpha^{2} / 4 K_{1}}=\frac{L \rho \alpha^{2}}{4},
$$

where $L, \rho$ are the latent heat and density of the material. (23), (24), (25), (27) and (28) determine $A, B, C, D$ and $\alpha$. Eliminating $A, B, C, D$, we have, for $\alpha$ :

$$
\frac{Q}{4 \pi} e^{-\alpha^{2} / 4 K_{2}}+\frac{k_{1} \Theta e^{-\alpha^{2} / 4 K_{1}}}{E i\left(-\alpha^{2} / 4 K_{1}\right)}=\frac{L \rho \alpha^{2}}{4} .
$$

The left side of (29) decreases monotonically from $Q / 4 \pi$ when $\alpha=0$ to $-\infty$ when $\alpha=\infty$. Hence the equation has always one, and only one, real positive root, $\alpha$ and the solution is :

$$
\begin{aligned}
& \theta_{2}=\frac{Q}{4 \pi k_{2}}\left[E i\left(-\alpha^{2} / 4 K_{2}\right)-E i\left(-r^{2} / 4 K_{2} t\right)\right], \quad 0<r<\alpha \sqrt{t} . \\
& \theta_{1}=-\Theta\left[1-\frac{E i\left(-r^{2} / 4 K_{1} t\right)}{E i\left(-\alpha^{2} / 4 K_{1}\right)}\right], \quad r>\alpha \sqrt{t} .
\end{aligned}
$$

As an example, consider an ice-water system, for which

Water : $k_{2}=0.00144 \mathrm{Cal} / \mathrm{cm}$. sec. $^{\circ} \mathrm{K} . \quad K_{2}=0.00144 \mathrm{~cm} . .^{2} / \mathrm{sec}$.

Ice : $k_{1}=0.0053 \mathrm{Cal} / \mathrm{cm}$. sec. ${ }^{\circ} \mathrm{K} . \quad K_{1}=0.0155 \mathrm{~cm} .{ }^{2} / \mathrm{sec}$.

and $L \rho$ (ice) $=73 \cdot 6 \mathrm{Cal} / \mathrm{cm}^{3}$

Suppose that $-\Theta=-2$, and $Q=2 \cdot 38 \mathrm{Cal} / \mathrm{cm}$. sec., which represents approximately the heat supplied by a very thin wire, of resistance $10 \Omega / \mathrm{cm}$, carrying a current of 1 amp. Then (29) yields

$$
\alpha \doteqdot 0 \cdot 06637 \mathrm{~cm} . / \mathrm{sec}^{\frac{1}{2}} \text {. }
$$

and $A=1 \cdot 681^{\circ}, B=2^{\circ}, C=131 \cdot 7^{\circ}, D=43 \cdot 73^{\circ}$.

The corresponding temperature profiles are shown in Table $I$., on page 45 .

In practice, since $\theta_{2}$ rises indefinitely, a boundary of vaporisation must follow the boundary of fusion. The theory could be extended to cover this if the effect of pressure on the boiling point and latent heat were ignored. Although this is not legitimate in the case described, the solution will be included for its general interest. If suffix 3 refers to the vapour state, we assume in addition to (20), (21) :

$$
\theta_{3}=-F E i\left(-r^{2} / 4 K_{3} t\right)-G
$$


Table I

$\begin{array}{ccc}r / \sqrt{t} & \theta_{2} & \theta_{1} \\ \left(\mathrm{~cm} . / \sec ^{\frac{1}{2}} .\right) & \left({ }^{\circ} \mathrm{C} .\right) & \left({ }^{\circ} \mathrm{C} .\right) \\ 0 \cdot 024 & 196 \cdot 6 & \\ 03394 & 117 \cdot 6 & \\ 04158 & 75 \cdot 56 & \\ 04799 & 48 \cdot 78 & \\ 05367 & 30 \cdot 00 & \\ 05879 & 16 \cdot 12 & \\ 06351 & 5 \cdot 51 & -0 \cdot 8196 \\ 06637 & 0 & -1 \cdot 2362 \\ 09248 & & -1 \cdot 4780 \\ 1128 & & -1 \cdot 6312 \\ 1302 & & -1 \cdot 8319 \\ 1456 & & -1.9178 \\ 1783 & & -1 \cdot 9581 \\ 2059 & & -1.9781 \\ 2302 & & -1 \cdot 9883 \\ 2523 & & -1 \cdot 9936 \\ 2724 & & \end{array}$

Then the boundaries of vaporisation and fusion are $r=\alpha_{12} \sqrt{t}, r=\alpha_{23} \sqrt{t}$, say, whereupon (23) and (24) apply, and also :

$$
\begin{aligned}
& C E i\left(-\alpha_{23}^{2} / 4 K_{2}\right)+D=-V, \\
& F E i\left(-\alpha_{23}^{2} / 4 K_{3}\right)+G=-V,
\end{aligned}
$$

where $V$ is the boiling point, referred to the melting point as zero. (25) holds as before, while $F$ and $k_{3}$ must be substituted for $C$ and $k_{2}$ in (27). (28) is replaced by two similar equations, involving $L_{12}$ and $L_{23}$. We have thus eight relations to determine $A, B, C, D, F, G, \alpha_{12}, \alpha_{23}$. Elimination of the six coefficients leads to the following simultaneous equations for $\alpha_{12}, \alpha_{23}$ :

$$
\begin{gathered}
\frac{Q}{4 \pi} e^{\frac{-\alpha_{23}^{2}}{4 K_{3}}}-\frac{k_{2} V e^{\frac{-\alpha_{23}^{2}}{4 K_{2}}}}{E i\left(-\frac{\alpha_{12}^{2}}{4 K_{2}}\right)-E i\left(-\frac{\alpha_{23}^{2}}{4 K_{2}}\right)}=\frac{L_{23} \rho}{4} \alpha_{23}^{2}, \\
\frac{k_{2} V e^{\frac{-\alpha_{12}^{2}}{4 K_{2}}}}{E i\left(\frac{\alpha_{12}^{2}}{-4 K_{2}}\right)-E i\left(-\frac{\alpha_{23}^{2}}{4 K_{2}}\right)}+\frac{k_{1} \Theta e^{\frac{-\alpha_{12}^{2}}{4 K_{1}}}}{E i\left(-\frac{\alpha_{12}^{2}}{4 K_{1}}\right)}=\frac{L_{12} \rho}{4} \alpha_{12}^{2} .
\end{gathered}
$$

These could be solved cyclically in a numerical case. This has not been carried out for the system ice-water-steam, in view of the physically unreal assumptions.

4. Propagation from a point source. Let $Q$ units of heat be generated per unit time at the origin, the conditions being otherwise as before. A spherical boundary $r=R(t)$ proceeds outward. We assume the solution :

$$
\begin{aligned}
& \theta_{1}=A\left[\frac{\sqrt{K_{1} t}}{r} e^{-r^{2} / 4 K_{1} t}-\frac{\sqrt{\pi}}{2} \operatorname{erfc} \frac{r}{2 \sqrt{K_{1} t}}\right]-B, \\
& \theta_{2}=C\left[\frac{\sqrt{K_{2} t}}{r} e^{-r^{2} / 4 K_{2} t}-\frac{\sqrt{\pi}}{2} \operatorname{erfc} \frac{r}{2 \sqrt{K_{2} t}}\right]-D .
\end{aligned}
$$


Then

say, and

$$
R(t)=\alpha \sqrt{t}
$$

$$
\begin{aligned}
& A\left[\frac{\sqrt{K_{1}}}{\alpha} e^{-\alpha^{2} / 4 K_{1}}-\frac{\sqrt{\pi}}{2} \operatorname{erfc} \frac{\alpha}{2 \sqrt{K_{1}}}\right]=B, \\
& C\left[\frac{\sqrt{K_{2}}}{\alpha} e^{-\alpha^{2} / 4 K_{2}}-\frac{\sqrt{\pi}}{2} \operatorname{erfc} \frac{\alpha}{2 \sqrt{K_{2}}}\right]=D .
\end{aligned}
$$

Also, as before,

$$
B=\Theta \text {, }
$$

while

$$
\frac{\partial \theta_{1}}{\partial r}=-\frac{A \sqrt{K_{1} t}}{r^{2}} e^{-r^{2} / 4 K_{1} t} ; \quad \frac{\partial \theta_{2}}{\partial r}=-\frac{C \sqrt{K_{2} t}}{r^{2}} e^{-r^{2} / 4 K_{2} t} .
$$

Thus

$$
\underset{r \rightarrow 0}{\mathrm{~L}}\left[-4 \pi k_{2} r^{2} \frac{\partial \theta_{2}}{\partial r}\right]=4 \pi k_{2} \sqrt{K_{2} t} . C
$$

so that $Q=q \sqrt{t}$, say, $q$ constant, and

$$
C=q / 4 \pi k_{2} \sqrt{K_{2}} .
$$

From (2), (39), (40), (42), (43), we obtain the following equation for $\alpha$ :

$$
\frac{q}{4 \pi} e^{-\alpha^{2} / 4 K_{2}}-\frac{k_{1} \alpha \Theta}{1-\frac{\alpha}{2} \sqrt{\frac{\pi}{K_{1}}} e^{\alpha^{3} / 4 K_{1}} \operatorname{erfc}\left(\frac{\alpha}{2 \sqrt{K_{1}}}\right)}=\frac{L \rho \alpha^{3}}{2} .
$$

The left side again decreases monotonically from $q / 4 \pi$ to $-\infty$ as $\alpha$ increases from 0 to $\infty$. Hence (44) has always one and only one real positive root. We have therefore derived a solution for propagation from a point source whose strength increases as $\sqrt{t}$.

The solution may be readily extended to cover the case in which two (or more) surfaces of phase-change arise. The analysis is similar to that indicated above for the cylindrical system.

Appendix. Proof that the functions in (29) and (44) decrease monotonically for $\alpha>0$.

Consider $f(x) \equiv e^{x} E i(-x)$,

$$
\frac{d f}{d x}=e^{x}\left[E i(-x)+e^{-x} / x\right] \equiv e^{x} g(x) \text { say }
$$

Then

$$
\frac{d g}{d x}=-e^{-x} / x^{2}<0
$$

But when $x=+\infty, g=0$. Hence $g>0$, and so $d f / d x>0$. It follows at once that the function on the left of (29) decreases monotonically. Its value is $Q / 4 \pi$ at 0 and $-\infty$ at $\infty$.

Again, let

Then

$$
\begin{aligned}
f(x) & \equiv \frac{1}{x}-\sqrt{\pi} e^{x^{2}} \operatorname{erfc}(x), \\
d f / d x & =-x e^{x^{2}}\left[2 \sqrt{\pi} \operatorname{erfc}(x)-\frac{2}{x} e^{-x^{2}}+\frac{1}{x^{3}} e^{-x^{2}}\right] \\
& \equiv-x e^{x^{2}} g(x), \text { say. }
\end{aligned}
$$

$$
d g / d x=-3 e^{-x^{2}} / x^{4}<0 .
$$


But

$$
g(\infty)=0 \text {. }
$$

Hence $g>0$ and so $d f / d x<0$. It follows at once that the function on the left of (44) decreases monotonically. Its value is $q / 4 \pi$ at 0 and $-\infty$ at $\infty$.

\section{REFERENCES}

(1) Stefan, Wied. Ann., 41, 725 (1890); 42, 269 (1891).

(2) Carslaw and Jaeger, Conduction of Heat in Solids, Oxford, 1947, pp. 71, 227.

Imperial Chemical Industries Ltd

Nobel Division Research Department

Stevenston 